\title{
A Secure Network Detection System against Noisy Unlabeled Data
}

\author{
Shailesh Kumar Gaikwad, Prof. Vijay Shah, Yogendra Kumar Jain \\ Department of Computer Science \& Engineering \\ Samrat Ashok Technological Institute \\ Vidisha, (M.P.), India
}

\begin{abstract}
Today, the Internet along with the corporate network plays a major role in creating and advancing new business avenues. With the ever increasing deployment and usage of gigabit networks, traditional network anomaly detection based intrusion detection systems have not scaled accordingly. Most, if not all, systems deployed assume the availability of complete and clean data for the purpose of intrusion detection. We contend that this assumption is not valid. Factors like noise in the audit data, mobility of the nodes, and the large amount of data generated by the network make it difficult to build a normal traffic profile of the network for the purpose of anomaly detection. From this perspective, the leitmotif of the research effort described in this dissertation is the design of a novel intrusion detection system that has the capability to detect intrusions with high accuracy even when complete audit data is not available. In this dissertation, we take a holistic approach to anomaly detection to address the threats posed by network based denial-of-service attacks by proposing improvements in every step of the intrusion detection process. At the data collection phase, we have implemented an adaptive sampling scheme that intelligently samples incoming network data to reduce the volume of traffic sampled, while maintaining the intrinsic characteristics of the network traffic. A Bloom filters based fast flow aggregation scheme is employed at the data pre-processing stage to further reduce the response time of the anomaly detection scheme. Lastly, this dissertation also proposes an expectation-maximization algorithm based anomaly detection scheme that uses the sampled audit data to detect intrusions in the incoming network traffic.
\end{abstract}

Keywords- Network Security, Anomaly Detection, Intrusion detection, Clustering.

\section{INTRODUCTION}

The aforementioned access points make today's networks more vulnerable to intrusions and attacks. Cyber-crime is no longer the prerogative of the stereotypical hacker. Joining ranks with the hackers are disgruntled employees, unethical corporations, and even terrorist organizations. With the vulnerability of present-day software and protocols combined with the increasing sophistication of attacks, it comes as no surprise that network based attacks are on the rise. The 2009 annual Computer Crime and Security survey [4], jointly conducted by the Computer Security Institute and the FBI. Although 86 percent of the respondents used firewalls, their consensus was that firewalls by themselves are not sufficient to provide adequate protection. Moreover, according to recent studies, an average of twenty to forty new vulnerabilities in commonly used networking and computer products are discovered every month. Such wide-spread vulnerabilities in software add to today's insecure computing/networking environment. This insecure environment has given rise to the ever evolving field of intrusion detection and prevention. The cyberspace's equivalent to the burglar alarm, intrusion detection systems complement the beleaguered firewall.

An intrusion detection system gathers and analyzes information from various areas within a computer or a network to identify possible security breaches. In other words, intrusion detection is the act of detecting actions that attempt to compromise the confidentiality, integrity or availability of a system/network. Traditionally, intrusion detection systems have been classified as signature detection systems, anomaly detection systems or a hybrid/ compound detection systems. A signature detection system identifies patterns of traffic or application data presumed to be malicious while anomaly detection systems compare activities against a "normal" baseline. On the other hand, a hybrid intrusion detection system combines the techniques of the two approaches. Both signature detection and anomaly detection systems have their share of advantages and drawbacks. The primary advantage of signature detection is that known attacks can be detected fairly reliably with a low false positive rate. The major drawback of the signature detection approach is that such systems typically require a signature to be defined for all of the possible attacks that an attacker may launch against a network. The biggest advantage of anomaly detection systems is that profiles of normal activity are customized for every system, application and/or network, and therefore making it very difficult for an attacker to know with certainty what activities it can carry out without getting detected. However, the anomaly detection approach also has its share of drawbacks: the intrinsic complexity of the system and the difficulty of associating alarms with the specific events that triggered those alarms [1] and [2].

However, most intrusion detection systems have not been able to keep up with the advances in high speed networking. Intrusion detection products, currently deployed in gigabit networks, need significant improvements before they can offer adequate protection against attacks. A majority of the products in the market today can detect less than half of the attacks directed at them, even though many of those attacks are well documented. Therefore the leitmotif of this dissertation is 
the development of a network based anomaly detection system that can detect intrusions/attacks in a large, high volume and high speed enterprise network.

\section{Intrusion Detection-}

An intrusion detection system is a software tool used to detect unauthorized access to a computer system or network. An intrusion detection system is capable of detecting all types of malicious network traffic and computer usage. This includes network attacks against vulnerable services, data driven attacks on applications, host-based attacks-such as privilege escalation, unauthorized logins and access to sensitive files-and malware. An intrusion detection system is a dynamic monitoring entity that complements the static monitoring abilities of a firewall. An intrusion detection system monitors traffic in a network in promiscuous mode, very much like a network sniffer. The network packets that are collected are analyzed for rule violations by a pattern recognition algorithm. When rule violations are detected, the intrusion detection system alerts the administrator. One of the earliest work that proposed intrusion detection by identifying abnormal behavior can be attributed to Anderson [5]. In his report, Anderson presents a threat model that classifies threats as external penetrations, internal penetrations, and misfeasance, and uses this classification to develop a security monitoring surveillance system based on detecting anomalies in user behavior. External penetrations are defined as intrusions that are carried out by unauthorized computer system users; internal penetrations are those that are carried out by authorized users who are not authorized for the data that is compromised; and misfeasance is defined as the misuse of authorized access both to the system and to its data [2].

In other words, a model of the behavior of the entity being monitored could be constructed by an intrusion detection system, and subsequent behavior of the entity could be verified against the entity's model. In this model, behavior that deviates sufficiently from the norm is considered anomalous. In the paper, Denning mentioned several models that are based on statistics, Markov chains, timeseries etc [3].

In a much cited survey on intrusion detection systems, put forth a generalized model of a typical intrusion detection system. Figure depicts such a system where solid arrows indicate data/control flow while dotted arrows indicate a response to intrusive activity. According to the generic architectural model of an intrusion detection system contains the following modules:

\section{- Audit data collection \\ - Audit data storage \\ - Analysis and detection \\ - Configuration data \\ - Reference data \\ - Active/processing data}

Alarm: This part of the system handles all output from the intrusion detection system. The output may be either an automated response to an intrusion or a suspicious activity alert for a system security officer. Historically, intrusion detection research has concentrated on the analysis and detection stage of the architectural model. As mentioned above, algorithms for the analysis and detection of intrusions/attacks are traditionally classified into the following three broad categories:

\section{i. $\quad$ Signature or misuse detection \\ ii. An anomaly detection system \\ iii. Statistical Anomaly Detection}

\section{Machine Learning based Anomaly Detection-}

Machine learning can be defined as the ability of a program and/or a system to learn and improve their performance on a certain task or group of tasks over time. Machine learning aims to answer many of the same questions as statistics or data mining. However unlike statistical approaches which tend to focus on understanding the process that generated the data, machine learning techniques focus on building a system that improves its performance with experience.

System Call and Sequence Analysis- One of the widely used machine learning echniques for anomaly detection involves learning the behavior of a program and recognizing significant deviations from the normal. In a seminal paper, Forrest et al. [4] established an analogy between the human immune system and intrusion detection. They did this by proposing a methodology that involved analyzing a program's system call sequences to build a normal profile. In their paper, they analyzed several UNIX based programs like sendmail, lpr etc. and showed that correlations in fixed length sequences of system calls could be used to build a normal profile of a program. Therefore, programs that show sequences that deviated from the normal sequence profile could then be considered to be victims of an attack. The system they developed was only used off-line using previously collected data and used a quite simple table-lookup algorithm to learn the profiles of programs. Their work was extended by Hofmeyr et al. [5], where they collected a database of normal behavior for each program of interest. Once a stable database is constructed for a given program in a particular environment, the database was then used to monitor the program's behavior. The sequences of system calls formed the set of normal patterns for the database, and sequences not found in the database indicated anomalies.

\section{Self-Similarity and Network Traffic-}

In the last decade, most of the studies on network traffic argued convincingly that Internet traffic is very far from being regular, and presents large variations in its throughput at all scales [5]. These studies have shown that Internet traffic exhibits characteristics such as selfsimilarity [6], multi-fractality [1], and long-range dependence [5], which implies that in all cases network traffic can vary significantly. In addition, given the highly 
variable nature of Internet traffic, anomaly based intrusion detection systems are raising alarms for many disruptions that are not attacks. The high rate of false positives is one of the major shortcomings of current IDS and the current evolution of Internet traffic with larger and larger variations among time continues to limit the efficiency of anomaly based IDS.

\section{Properties of Network Traffic-}

A network intrusion detection must distinguish between hostile and benign traffic, and must do so quickly to keep up with a high speed network. Depending on whether the intrusion detection system uses signature or anomaly detection, it must either model attacks (of which there are thousands) or normal traffic. There are two main challenges for modeling normal traffic for anomaly detection. First, network traffic is very complex and unpredictable, and second, the model changes over time.

Wide area network traffic contains a wide range of anomalies and uncharacteristic data that cannot be easily explained for. Examples include private IP addresses, storms of packets routed in a loop until their TTLs expire, TCP acknowledgments of packets never sent, TCP retransmissions with inconsistent payloads, SYN packets with urgent data, and so on. ICMP packets with invalid code fields, and packets addressed to nonexistent hosts and ports. Many of these were investigated and found to be not hostile. Instead, many of the aforementioned errors were caused by mis-configured routers and/or DNS servers.

As a result, a confounding problem that has been faced by researchers in the field of Internet traffic modeling, is that it is not possible to determine the average rate of many types of events (for example, bytes per second or packets per second for some packet type), regardless of the duration of the sampling period. The long held paradigm in the communication and performance analysis communities has been that voice traffic and, by extension, data traffic could be modeled as a Markovian (e.g. Poisson) process, which could then be accurately analyzed and efficiently controlled. Therefore, if data traffic were to follow Markovian arrival process, it would have a characteristic burst length which would tend to be smoothed by averaging over a long enough time scale. However, in the mid nineties, it was discovered that this was not the case. In a seminal paper, Leland et al. [7] discovered that measurements on real Internet traffic traces indicated that a significant traffic variance (burstiness) is present on a wide range of time scales. It was found that if we graphed packets per second or packets per month, they would both show bursts of high traffic rates separated by gaps of low activity. Furthermore, both graphs would look the same. A burst or gap could last for a fraction of a second or for months. The distribution of traffic rates would be independent of time scale. This behavior was akin to the behavior displayed by self-similar or fractal processes.

\section{PROPOSED SAMPLING ALGORITHM}

Traffic measurement and monitoring serves as the basis for a wide range of IP network operations and engineering tasks such as trouble shooting, accounting and usage profiling, routing weight configuration, load balancing, capacity planning, etc. Traditionally, traffic measurement and monitoring is done by capturing every packet traversing a router interface or a link. With today's highspeed links, such an approach is no longer feasible due to the excessive overheads it incurs on line-cards or routers. As a result, packet sampling has been suggested as a scalable alternative to address this problem.

We describe the weighted least squares predictor that is utilized for predicting the next sampling interval. This predictor has been adopted because of its capability to follow the trends in network traffic.

\section{Weighted Least Square Predictor-}

Let us assume that the vector $\mathrm{Z}$ holds the values of the $N$ previous samples, such that $Z N$ is the most recent sample and $Z 1$ is the oldest sample. Having fixed a window size of $N$, when the next sampling occurs, the vector is right shifted such that $Z N$ replaces $Z N-1$ and $Z 1$ is discarded. The weighted prediction model therefore predicts the value of $Z N$ given $Z N-1 ; \ldots ; Z 1$. In general, we can express this predicted value as a function of the $N$ past samples i.e.,

$$
Z N=\alpha^{\mathrm{T}} Z \text {, }
$$

where $Z^{\prime} N$ is the new predicted value, $Z$ is the vector of past $N-1$ samples, and $\alpha^{\mathrm{T}}$ is a vector of predictor coefficients distributed such that newer values have a greater impact on the predicted value $Z^{\prime} N$. A second vector, $t$, records the time that each sample is taken and is shifted in the same manner as Z. The objective of the weighted prediction algorithm is to find an appropriate coefficient vector, $\alpha^{\mathrm{T}}$ such that the following summation is minimized

$$
\mathrm{S}=\sum_{\mathrm{i}=0}^{\mathrm{n}-1} \mathrm{Wi}\left(\mathrm{Zi}-\mathrm{Z}^{\prime}\right)^{2}
$$

where $i, Z i$, and $Z^{\prime}$ denote the weight, the actual sampled value, and the predicted value in the ith interval, respectively.

The coefficient vector is given by:

$$
\alpha^{\mathrm{T}}=\left(\mathrm{Z}^{\mathrm{T}} \mathrm{WZ}\right)^{-1} \mathrm{Z}^{\mathrm{T}} \mathrm{W}
$$

where $\mathrm{W}=\mathrm{W}^{\mathrm{T}} \mathrm{W}$ is a $(N-1) \mathrm{X}(N-1)$ diagonal weight matrix and $\mathrm{w}$ is a $N X 1$ weight vector with weight co-efficient $w I$ that are determined according to two criteria:

1. The "freshness" of the past $N$ - 1 samples. A more recent sample has a greater weight.

2. The similarity between the predicted value at the beginning of the time interval and the actual value. The similarity between the two values is measured by the distance between them. The smaller the Euclidean distance is, the more similar they are to each other.

\section{Adaptive Weighted Sampling-}

Adaptive sampling algorithms dynamically adjust the sampling rate based on the observed sampled data. A key element in adaptive sampling is the prediction of future 
behavior based on the observed samples. The weighted sampling algorithm described utilizes the weighted least squares predictor to select the next sampling interval. Inaccurate predictions by the weighted least squares predictor indicates a change in the network traffic behavior and requires a change in the sampling rate.

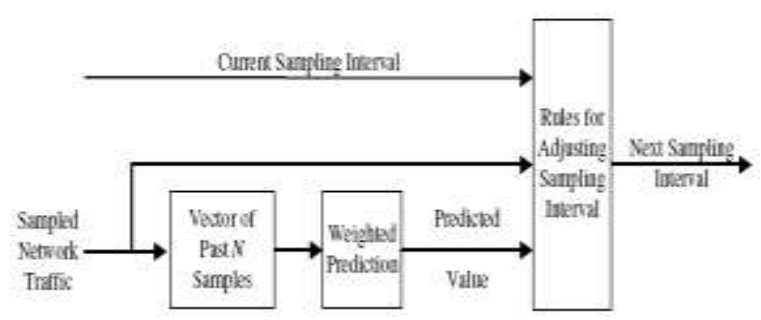

Figure: Block diagram of the adaptive sampling algorithm

The proposed adaptive sampling algorithm consists of the following steps :

1. Fix the first $N$ sampling intervals. (In our simulations we used $\mu=60 \mathrm{sec}$. and $N=10$ )

2. Apply the weighted least squares predictor to predict the anticipated value, $Z$ ' $N$, of the network parameter.

3. Calculate the network parameter value at the end of the sampling time period.

4. Compare the predicted value with the actual value.

5. Adjust sampling rate according to the predefined rule set if the predicted value differs from the actual value.

My System- To achieve the goal of detecting intrusions in high bandwidth environments, SCAN's architecture combines intelligent sampling and flow aggregation with data reduction and anomaly detection to achieve a high degree of accuracy in detecting intrusions with partial audit data. The design requirements for such a network based IDS were (a) stateless inspection of packets, protocols and/or packet headers at wire speed, (b) low occurrence of false alarms and high detection rate, (c) ability to track TCP states, and (d) ability to report events and/or alarms. Based on these requirements is composed of the following modules:

\section{- $\quad$ Adaptive weighted packet sampling module \\ - Flow aggregation module \\ - Data reduction module \\ - Clustering And Anomaly detection module}

involved evaluating the components of SCAN individually before combining them and testing the system as a whole. In this direction, we first evaluated the performance of the proposed

sampling algorithm. By comparing its performance with the simple random sampling algorithm. We illustrated, through our simulation based analysis that the proposed sampling algorithm is superior to the traditionally used simple random sampling algorithm in predicting the packet mean delay while reducing the volume of traffic and maintaining the self similarity of the original network flows.

The second step of the evaluation process involves evaluating the proposed EM algorithm based clustering scheme. To do this, we compared the performance of the proposed algorithm with the widely used $k$-means clustering algorithm. Results illustrated that not only is more accurate, but the anomalous flow algorithm has a better performance when the clustering algorithm that is employed.

Lastly, we evaluated the performance of the anomalous flow detection algorithm. Our simulation results show that even with $10 \%$ missing data, the accuracy of clustering is in the high eighties. We also show, through our simulations, that the rate of detection with up to $17 \%$ missing data is very high as well.

Ample evidence of the efficient and robust performance of the proposed anomaly detection based intrusion detection system. The results have vidicated our assertion that parametric estimation techniques like the EM algorithm can be used effectively to detect intrusions in high bandwidth networks by employing sampling based approaches.

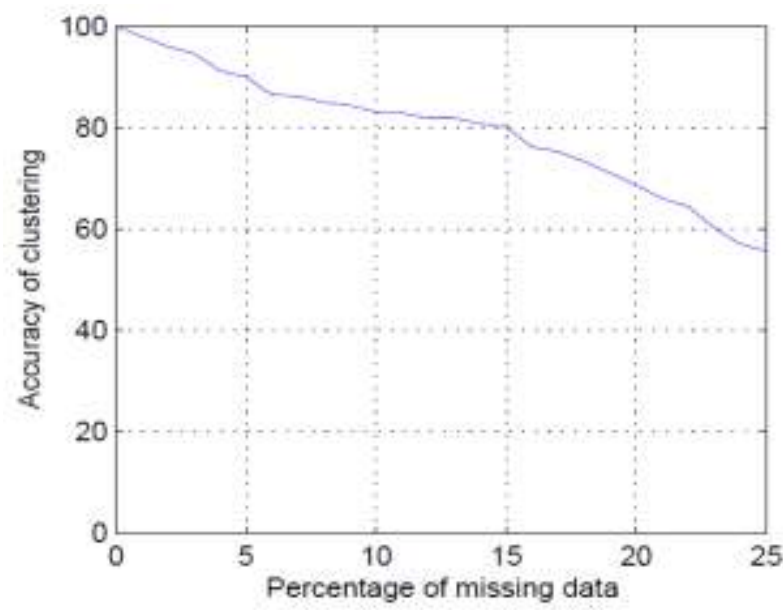

Figure: Accuracy of clustering with POTION vs. percentage of missing data.

\section{CONCLUSION}

This has presented conclusions based upon the research results, and recommended areas of future research. The goal of this research was to provide a methodology to detect network based attacks with incomplete audit data. The proposed scheme attempts to fill a niche in the
The results from the simulation based analysis that was performed on SCAN. The evaluation methodology 
intrusion detection domain, by attempting to address the problem of detecting network based denial-of-service attacks in high performance, high availability and high speed networks. By employing an intelligent sampling scheme reduces the computational complexity by reducing the volume of audit data that is processed without losing the intrinsic characteristics of the network traffic. In addition, employs an improved Expectation-Maximization algorithm based clustering technique to impute the missing values and further increase the accuracy of anomaly detection.

\section{REFERENCES}

[1]Abdul Samad bin Haji Ismail, Abdul Hanan Abdullah, Kamalrulnizam bin Abu Bak, "A Novel Method for Unsupervised Anomaly Detection using Unlabelled Data" IEEE2008.

[2] Nam Hun Park, Won Suk Lee," Anomaly Detection over Clustering Multi-dimensional Transactional Audit Streams" IEEE2008.

[3] C. C. Aggarwal and P. S. Yu, "Outlier detection for high dimensional data," in Proceedings of the 2001 ACM SIGMOD international conference on Management of data, T. Sellis, Ed. ACM Press, May 2001, pp. $37-46$.

[4] R. Agrawal, T. Imielinski, and A. Swami, "Mining association rules between sets of items in large databases," in In Proceedings of the ACM SIGMOD Conference on Management of Data. ACM Press, 1993, pp. 207-216.
[5] D. Anderson, T. Frivold, A. Tamaru, and A. Valdes, "Next generation intrusion detection expert system (nides), software users manual," Computer Science Laboratory, SRI International, 333 Ravenswood Avenue, Menlo Park, CA 94025-3493, User Manual SRI-CSL-95-07, 1994.

[6] D. Anderson, T. F. Lunt, H. S. Javitz, A. Tamaru, and A. Valdes, "Detecting unusual program behavior using the statistical component of the next-generation intrusion detection expert system (nides)," Computer Science Laboratory, SRI International, 333 Ravenswood Avenue, Menlo Park, CA 94025-3493, Technical Report SRICSL- 95-06, May 1995.

[7] J. P. Anderson, "Computer security threat monitoring and surveillance," James P. Anderson Co., Fort Washington, Pennsylvania, Technical Report, April 1980.

[8] S. Axelsson, "Research in intrusion-detection systems: A survey," In http:// citeseer.ist.psu.edu/axelsson98research.html, Department of Computer Engineering, Chalmers University of Technology, Technical Report 98-17, December 1998. 